\title{
Algoritmos Rápidos para Cifragem de Imagens Utilizando Aproximações da DCT de Comprimento $8^{*}$
}

\author{
Thiago L. T. da Silveira ${ }^{\dagger}$ \\ Universidade Federal de Santa Maria \\ Depto de Eletrônica e Computação e LACESM \\ E-mail: thiago@inf.ufsm.br \\ Renato J. Cintra \\ Universidade Federal de Pernambuco \\ Grupo de Processamento de Sinais, Depto de Estatística \\ E-mail: rjdsc@stat.ufpe.org
}

\author{
Fábio M. Bayer \\ Universidade Federal de Santa Maria \\ Depto de Estatística e LACESM \\ E-mail: bayer@ufsm.br \\ Alice J. Kozakevicius \\ Universidade Federal de Santa Maria \\ Depto de Matemática e LANA \\ E-mail: alice.kozakevicius@gmail.com
}

\section{$\underline{\text { RESUMO }}$}

\section{Introdução}

Criptografia é o estudo de técnicas matemáticas relacionadas a aspectos de segurança da informação, tais como confidencialidade, integridade e autenticidade de dados [4]. Vários processos de cifragem de imagens envolvem transformadas discretas, como a transformada discreta de Fourier [5], a transformada discreta de Hartley (DHT) [2] e, recentemente, a DHT binária (BDHT) [1]. A BDHT é uma aproximação de baixa complexidade computacional para a DHT e sua utilização em cifragem de imagens torna o método rápido e eficiente.

Seguindo o método de codificação de dupla fase aleatória introduzido por [5] e suportada por [1], este trabalho propõem uma investigação de alternativas rápidas e eficientes para cifragem de imagens com três contribuições distintas: (i) utilização da transformada discreta do cosseno (DCT) no algoritmo de cifragem; (ii) utilização de aproximações da DCT de complexidade multiplicativa nula; e (iii) consideração de decomposição da imagem original em sub-blocos, assim como em algoritmos de compressão de imagens, como JPEG. Os pontos (ii) e (iii) são objetivados com o intuito de reduzir o custo computacional envolvido no procedimento de cifragem, propondo algoritmos rápidos e eficientes para aplicações em tempo real.

\section{Metodologia}

Os métodos de cifragem e decifragem de imagens propostos em [1] podem ser descritos, respectivamente, por

$$
\begin{aligned}
& \mathbf{Q}=\mathbf{T}_{N}^{\top} \cdot\left(\left(\mathbf{T}_{N} \cdot\left(\mathbf{P} \odot \mathbf{K}_{1}\right) \cdot \mathbf{T}_{N}^{\top}\right) \odot \mathbf{K}_{2}\right) \cdot \mathbf{T}_{N}, \\
& \mathbf{R}=\left(\mathbf{T}_{N}^{\top} \cdot\left(\left(\mathbf{T}_{N} \cdot \mathbf{Q} \cdot \mathbf{T}_{N}^{\top}\right) \oslash \mathbf{K}_{2}^{\prime}\right) \cdot \mathbf{T}_{N}\right) \oslash \mathbf{K}_{1}^{\prime},
\end{aligned}
$$

em que $\mathbf{T}_{N}$ é a matriz $N \times N$ da BDHT, $\mathbf{P}$ é a imagem original de tamanho $N \times N$, $\mathbf{Q}$ é a imagem cifrada, $\mathbf{R}$ é a imagem decifrada, $\mathbf{K}_{1}, \mathbf{K}_{1}^{\prime}, \mathbf{K}_{2}$ e $\mathbf{K}_{2}^{\prime}$ são matrizes $N \times N$ aleatórias chamadas de chave, $\odot$ é a operação de multiplicação elemento-a-elemento e $\oslash$ é a operação de divisão elemento-a-elemento. Se $\mathbf{K}_{1}^{\prime}$ e $\mathbf{K}_{2}^{\prime}$ forem iguais, respectivamente, a $\mathbf{K}_{1}$ e $\mathbf{K}_{2}$ então a imagem $\mathbf{R}$ será igual a imagem original $\mathbf{P}$.

\footnotetext{
*Este trabalho foi parcialmente financiado por CNPq, FACEPE e FIT/UFSM.

${ }^{\dagger}$ Bolsista de Iniciação Tecnológica FIT/UFSM.
} 
Propomos a substituição da BDHT bidimensional (2D) por outras transformadas 2D como a DCT, a DCT binária (BDCT) [1] e a clássica transformada de Walsh-Hadamard (WHT). Além disso, sugerimos o procedimento de divisão da imagem original em sub-blocos de tamanho $k \times k$, para $k=8,16,32,64$. Em contraste, em [1], são utilizadas transformadas com mesmas dimensões das imagens. A Figura 1 esquematiza graficamente o método de cifragem considerado.

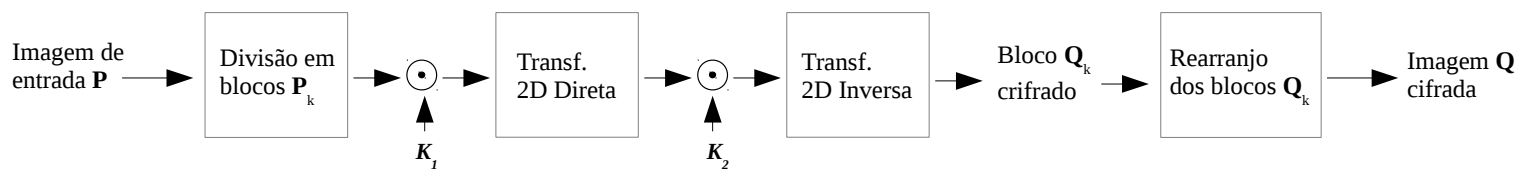

(a) Cifragem

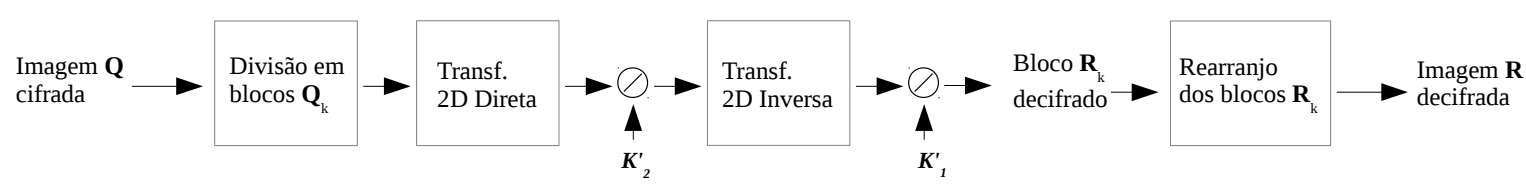

(b) Decifragem

Figura 1: Esquema de cifragem e decifragem de imagens.

\section{Resultados e discussões}

Uma vantagem direta da aplicação do método de sub-divisão da imagem original para o processo de cifragem é a redução do custo computacional. O custo computacional é avaliado pela complexidade aritmética que é dada pelo número de multiplicações e adições exigidas pela aplicação deste método. Algoritmos rápidos para transformadas discretas são comumente comparados em termos de sua complexidade aritmética, como figura de mérito para eficiência. A Tabela 1 apresenta as complexidades aritméticas associadas aos algoritmos de cifragem considerados. As complexidades da DCT exata de comprimento $k, \mathrm{DCT}_{k}$, são determinadas por meio do algoritmo de Chen [3] e as imagens utilizadas em nosso experimentos são imagens $512 \times 512$ em escala de cinza de 8 bits.

Tabela 1: Comparação da complexidade aritmética dos algoritmos considerados

\begin{tabular}{l|c|rrr|rrr}
\hline $\begin{array}{l}\text { Transformada } \\
\text { considerada }\end{array}$ & Número de & \multicolumn{2}{|c|}{ Complexidade de cada sub-bloco } & \multicolumn{3}{|c}{ Complexidade total } \\
sub-blocos & Mult. & Adições & Total & Mult. & Adições & Total \\
\hline DCT $_{512}$ & 1 & 3936256 & 6293504 & 10229760 & 3936256 & 6293504 & 10229760 \\
DCT $_{64}$ & 64 & 37376 & 61696 & 99072 & 2392064 & 3948544 & 6340608 \\
DCT $_{32}$ & 256 & 7424 & 12416 & 19840 & 1900544 & 3178496 & 5079040 \\
DCT $_{16}$ & 1024 & 1408 & 2368 & 3776 & 1441792 & 2424832 & 3866624 \\
DCT $_{8}$ & 4096 & 256 & 416 & 672 & 1048576 & 1703936 & 2752512 \\
HHT $_{8}$ & 4096 & 0 & 384 & 384 & 0 & 1572864 & 1572864 \\
BDCT $_{8}$ & 4096 & 0 & 384 & 384 & 0 & 1572864 & 1572864 \\
\hline
\end{tabular}

Os resultados da Tabela 1 evidenciam que quanto menor o tamanho do bloco, menor é a complexidade aritmética do algoritmo de cifragem associado. Os resultados do experimento computacional para comparação dos desempenhos dos procedimentos de cifragem considerando a DCT com diferentes tamanhos de sub-blocos são apresentados na Figura 2. Essa figura apresenta o índice de similaridade estrutural (MSSIM) [6] entre as imagens decifradas e a imagem original. A medida MSSIM varia no intervalo $[0,1]$ e considera características do sistema visual humano diferentemente das medidas usuais, tais como a relação sinal-ruído de pico e o erro quadrático médio [6]. Para essa aplicação, os $(i, j)$-ésimos elementos de $\mathbf{K}_{1}^{\prime}$ e $\mathbf{K}_{2}^{\prime}$ foram definidos como $\left[\mathbf{K}_{1}^{\prime}\right]_{i, j}=\left[\mathbf{K}_{1}\right]_{i, j}+\delta$ e $\left[\mathbf{K}_{2}^{\prime}\right]_{i, j}=\left[\mathbf{K}_{2}\right]_{i, j}+\delta$, em que os elementos $\left[\mathbf{K}_{1}\right]_{i, j}$ e $\left[\mathbf{K}_{2}\right]_{i, j}$ são independentes e uniformemente distribuídos em $[-0.001 ; 0.001]$ e $-0.001 \leq \delta \leq 0.001$. Para $k=8$, o gráfico com os valores de MSSIM decai mais rapidamente à medida que $\delta$ se afasta de zero, indicando que são necessárias chaves $\mathbf{K}^{\prime}{ }_{1}$ e $\mathbf{K}_{2}^{\prime}$ com valores mais próximos de $\mathbf{K}_{1}$ e $\mathbf{K}_{2}$, respectivamente, para a boa reconstrução da imagem original. Ou seja, a abordagem em sub-blocos, além de fornecer menor custo, também mostra-se como uma opção mais segura para cifragem de imagens. 


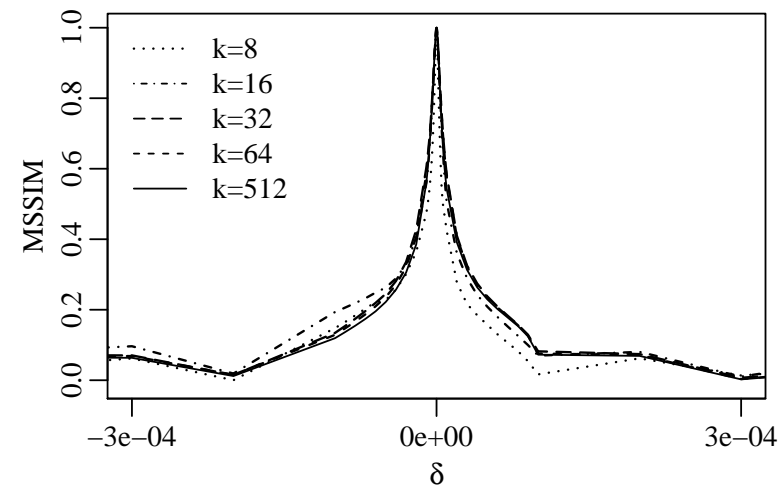

Figura 2: MSSIM das imagens decifradas utilizando a $\mathrm{DCT}_{k}, \operatorname{com} k=8,16,31,64$ e 512 .

Com base nos resultados anteriores, destacando o bom desempenho da utilização de $k=$ 8, optou-se por utilizar aproximações da DCT de complexidade multiplicativa nula, conforme Tabela 1, nomeadamente a WHT e a BDCT, como substituição da DCT de comprimento 8. A Figura 3 ilustra qualitativamente os resultados da cifragem por meio de alguns dos algoritmos rápidos considerados.

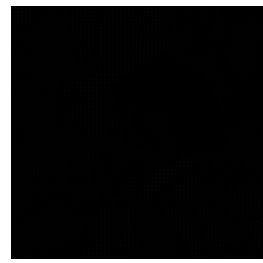

(a) $\mathrm{WHT}_{8}$

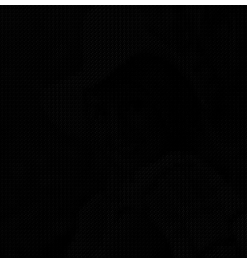

(b) $\mathrm{WHT}_{8}$

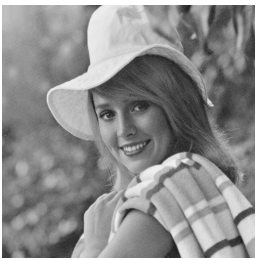

(c) $\mathrm{WHT}_{8}$

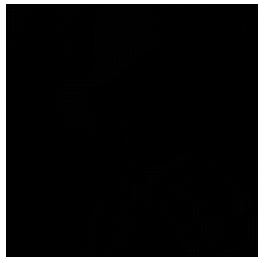

(d) $\mathrm{BDCT}_{8}$

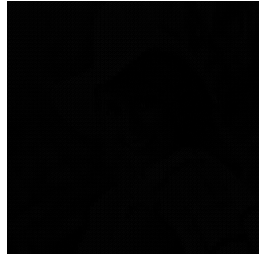

(e) $\mathrm{BDCT}_{8}$

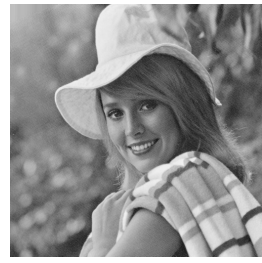

(f) $\mathrm{BDCT}_{8}$

Figura 3: Resultados da cifragem na imagem Elaine utilizando $\mathrm{WHT}_{8}(\mathrm{a}, \mathrm{b}, \mathrm{c})$ e $\mathrm{BDCT}_{8}$ (c,d,e): (a) cifrada, (b) decifrada com $\delta=0.001$, (c) decifrada com chaves corretas $(\delta=0)$, (d) cifrada, (e) decifrada $\operatorname{com} \delta=0.001$, (f) decifrada com chaves corretas $(\delta=0)$.

O procedimento de sub-divisão da imagem original para utilização de transformadas de menores comprimentos é bastante promissor. A utilização de transformadas aproximadas para a DCT de comprimento 8 resulta em algoritmos rápidos e úteis para aplicações em tempo real, ao mesmo tempo que mantém a segurança da imagem cifrada. Em trabalho futuro, serão investigadas transformadas discretas específicas para cifragem e a utilização de transformadas de baixa complexidade computacional de outros comprimentos.

Palavras-chave: Cifragem de imagens, DCT aproximada, Processamento de imagens

\section{Referências}

[1] S. Bouguezel, M. Ahmad, And M. Swamy, Binary discrete cosine and Hartley transforms, IEEE Transactions on Circuits and Systems I: Regular Papers, 60 (2013), pp. 989-1002.

[2] L. Chen And D. ZhaO, Optical image encryption with Hartley transforms, Optics Letters, 31 (2006), pp. $3438-3440$.

[3] W. H. Chen, C. Smith, And S. Fralick, A fast computational algorithm for the discrete cosine transform, IEEE Transactions on Communications, 25 (1977), pp. 1004-1009.

[4] A. J. Menezes, P. C. van Oorschot, and S. A. Vanstone, Handbook of Applied Cryptography, CRC Press, 5 ed., 2001.

[5] S.-C. PEI AND W.-L. Hsue, The multiple-parameter discrete fractional Fourier transform, IEEE Signal Processing Letters, 13 (2006), pp. 329-332.

[6] Z. Wang, A. C. Bovik, H. R. Sheikh, And E. P. Simoncelli, Image quality assessment: from error visibility to structural similarity, IEEE Transactions Image Processing, 13 (2004), pp. 600-612. 